\title{
A randomized controlled trial of light versus deep propofol sedation for elective outpatient colonoscopy: recall, procedural conditions, and recovery
}

\section{Une étude randomisée contrôlée sur la sédation légère $v s$ profonde au propofol pour la colonoscopie non urgente en ambulatoire: le souvenir, les conditions entourant l'intervention et la récupération}

\author{
Megan Allen, MClinEpi - Kate Leslie, MBBS, MD • Geoffrey Hebbard, MBBS, PhD • \\ Ian Jones, MBBS • Tejinder Mettho, MBBS • Paul Maruff, PhD
}

Received: 19 March 2015/Accepted: 13 August 2015/Published online: 3 September 2015

(C) Canadian Anesthesiologists' Society 2015

\begin{abstract}
Purpose This study aimed to determine if the incidence of recall was equivalent between light and deep sedation for colonoscopy. Secondary analysis included complications, patient clinical recovery, and postprocedure cognitive impairment.

Methods Two hundred patients undergoing elective outpatient colonoscopy were randomized to light (bispectral index [BIS] 70-80) or deep $(B I S<60)$ sedation with propofol and fentanyl. Recall was assessed
\end{abstract}

This article is accompanied by an editorial. Please see Can J Anesth 2015; 62: this issue.

Author contributions Megan Allen, Kate Leslie, Geoffrey Hebbard, Ian Jones, and Tejinder Mettho contributed substantially to the acquisition of data. Megan Allen, Kate Leslie, and Paul Maruff contributed substantially to the conception and design of the manuscript and to the analysis and interpretation of data. Megan Allen and Kate Leslie drafted the manuscript. Megan Allen, Kate Leslie, Geoffrey Hebbard, Ian Jones, Tejinder Mettho, and Paul Maruff reviewed the final manuscript.

M. Allen, MClinEpi $(\bowtie) \cdot K$. Leslie, MBBS, MD .

T. Mettho, MBBS

Department of Anaesthesia and Pain Management, Royal

Melbourne Hospital, Melbourne, Australia

e-mail: meganliseallen@gmail.com

M. Allen, MClinEpi - K. Leslie, MBBS, MD .

T. Mettho, MBBS

Anaesthesia, Perioperative and Pain Medicine Unit, University

of Melbourne, Melbourne, Australia

K. Leslie, MBBS, MD

Department of Pharmacology, University of Melbourne,

Melbourne, Australia by the modified Brice questionnaire, and cognition at baseline and discharge was assessed using a Cogstate test battery.

Results The median (interquartile range [IQR]) BIS values were different in the two groups (69 [65-74] light sedation vs 53 [46-59] deep sedation; $P<0.0001)$. The incidence of recall was $12 \%$ in the light sedation group and $1 \%$ in the deep sedation group. The risk difference for recall was 0.11 (90\% confidence interval, 0.05 to 0.17 ) in the intention-to-treat analysis, thus refuting equivalence in recall between light and deep sedation (0.05 significance level; $10 \%$ equivalence margin). Overall sedation-related complications were more frequent with deep sedation than with light sedation ( $66 \%$ vs $47 \%$, respectively; $P=0.008$ ). Recovery was more rapid with light sedation than with deep sedation as determined by the mean (SD) time to reach a score of 5 on the Modified Observer's Assessment of Alertness/Sedation Scale [3 (4) min vs 7 (4) min, respectively; $P<0.001]$ and by the median $[I Q R]$ time to

K. Leslie, MBBS, MD

Department of Epidemiology and Preventive Medicine, Monash

University, Melbourne, Australia

G. Hebbard, MBBS, PhD

Department of Gastroenterology, Royal Melbourne Hospital, Melbourne, Australia

G. Hebbard, MBBS, PhD

Department of Medicine, University of Melbourne, Melbourne, Australia 
readiness for hospital discharge (65 [57-80] min vs 74 [6386] min, respectively; $P=0.001)$. The incidence of postprocedural cognitive impairment was similar in those randomized to light (19\%) vs deep (16\%) sedation $(P=$ 0.554).

Conclusion Light sedation was not equivalent to deep sedation for procedural recall, the spectrum of complications, or recovery times. This study provides evidence to inform discussions with patients about sedation for colonoscopy. This trial was registered at the Australian and New Zealand Clinical Trials Registry, number 12611000320954.

\section{Résumé}

Objectif L'objectif de cette étude était de déterminer si l'incidence de souvenir était équivalente entre une sédation légère et une sédation profonde pour une colonoscopie. Nos analyses secondaires comprenaient les complications, la récupération clinique des patients, et les troubles cognitifs survenant après l'intervention.

Méthode Deux cents patients subissant une colonoscopie non urgente en clinique ambulatoire ont été randomisés à recevoir une sédation légère (indice bispectral [BIS] 70-80) ou profonde $(B I S<60)$ à base de propofol et de fentanyl. Le souvenir a été évalué à l'aide du questionnaire de Brice modifié, et la cognition au moment de l'accueil et du congé a été évaluée à l'aide de la batterie de tests abrégée Cogstate.

Résultats Les valeurs médianes (écart interquartile [ÉIQ]) de BIS étaient différentes dans les deux groupes (69 [65-74] sédation légère vs 53 [46-59] sédation profonde; $P<0,0001)$. L'incidence de souvenir était de $12 \%$ dans le groupe sédation légère et de $1 \%$ dans le groupe sédation profonde. La différence de risque de souvenir était de 0,11 (intervalle de confiance $90 \%, 0,05$ à $0,17)$ dans l'analyse selon l'intention de traiter, réfutant ainsi toute équivalence en matière de souvenir entre une sédation légère et une sédation profonde (seuil de signification 0,05; marge d'équivalence $10 \%$ ). Les

I. Jones, MBBS

Colorectal Surgery Unit, Royal Melbourne Hospital, Melbourne, Australia

I. Jones, MBBS

Department of Surgery, University of Melbourne, Melbourne, Australia

P. Maruff, $\mathrm{PhD}$

Cogstate Ltd, Melbourne, Australia

P. Maruff, $\mathrm{PhD}$

Centre for Neuroscience, University of Melbourne, Melbourne, Australia complications globales liées à la sédation étaient plus fréquentes dans le groupe sédation profonde que dans le groupe sédation légère (66 \% vs $47 \%$, respectivement; $P=0,008)$. La récupération a été plus rapide en cas de sédation légère qu'avec la sédation profonde telle que déterminée par le temps moyen (ÉT) pour atteindre une note de 5 sur l'échelle modifiée d'éveil/sédation selon l'évaluation de l'observateur (Modified Observer's Assessment of Alertness/Sedation Scale) [3 (4) min vs 7 (4) min, respectivement; $P<0,001]$ et par le temps médian [ÉIQ] nécessaire avant d'être prêt au congé de l'hôpital (65 [57-80] $\min$ vs 74 [63-86] min, respectivement; $P=0.001)$. L'incidence des troubles cognitifs post-procéduraux était semblable chez les personnes randomisées à recevoir une sédation légère $(19 \%)$ ou profonde $(16 \%)(P=0,554)$.

Conclusion La sédation légère n'était pas équivalente à la sédation profonde en matière de souvenir de l'intervention, du spectre de complications ou des temps de récupération. Cette étude procure des données probantes nécessaires pour informer les patients concernant la sédation pour la colonoscopie. Cette étude a été enregistrée au Registre australien et néozélandais des études cliniques, no. 12611000320954.

No consensus exists regarding the optimal depth of sedation for colonoscopy, with preferences among countries, regions, healthcare facilities, endoscopists, sedation providers, and patients ranging from no sedation at all to intentional general anesthesia. Patients often describe optimal sedation as amnesia for the procedure. A recent survey of patients awaiting colonoscopy indicated that awareness during the procedure was of greater concern than vomiting, prolonged drowsiness, respiratory complications, and even an incomplete colonoscopy examination. ${ }^{1}$ Other studies found that fear of intraprocedural pain and embarrassment were significant barriers to compliance with a colonoscopy examination. $^{2,3}$ In contrast, healthcare professionals may prioritize safety, ease of colonoscopy completion, and rapid and complete cognitive recovery to facilitate early discharge. Arguably, both lighter and deeper sedation can potentially achieve these goals.

Drug-induced hypnosis occurs on a continuum from mild drowsiness to deep unconsciousness, with the threshold for loss of recall occurring at a lighter depth of sedation than the lack of response to command. ${ }^{4}$ Consequently, even 'light' sedation may result in procedural amnesia despite clinical sedation scores showing preserved responsiveness. ${ }^{5}$ It is possible, therefore, that the incidence of recall may be equivalent between light and deep sedation protocols. ${ }^{5,6}$ Nevertheless, 
studies are lacking that make a direct comparison between light and deep sedation (using the same drugs) for colonoscopy with an objective measure of sedation depth, a validated assessment of post-procedure cognitive impairment, and measurements of complications and patient satisfaction. A lack of direct comparisons such as this impair the ability to advise patients about the advantages and disadvantages of different sedation depths, including the likelihood of procedural recall.

We therefore investigated whether light (bispectral index [BIS] 70-80) and deep (BIS < 60) propofol and fentanyl-based sedation for adult patients presenting for elective outpatient colonoscopy were equivalent with respect to the primary outcome of procedural recall. Secondary outcomes examined included complications, patient and anesthesiologist satisfaction with sedation, endoscopist ease of completion, as well as patient recovery profiles and post-procedure cognitive impairment.

\section{Methods}

With Institutional Ethics Committee approval (Melbourne Health, Aug 2011; 2011.066), adult patients scheduled for elective outpatient colonoscopy under sedation administered by an anesthesiologist at the Royal Melbourne Hospital were screened for eligibility. Written informed consent was obtained from eligible patients. Inclusion criteria were patients who were 18 years of age or older and American Society of Anesthesiologists' (ASA) physical status I-III. Exclusion criteria were patients undergoing an emergency or inpatient colonoscopy or other endoscopic procedures in addition to colonoscopy as well as those with cognitive, psychiatric, or language barriers to obtaining consent.

The primary outcome of recall was assessed in the post anesthesia care unit (PACU) using the modified Brice questionnaire. $^{7,8}$ The questionnaire was administered in the PACU once the patient had returned to full alertness (i.e., a score of 5 on the Modified Observer's Assessment of Alertness/Sedation [MOAA/S] $)^{9}$ following sedation. Patient satisfaction with sedation was scored on a fivepoint Likert scale ( $1=$ very dissatisfied; $5=$ very satisfied). The ease of colonoscopy completion was rated by the endoscopist on an 11-point Likert scale $(0=$ very difficult; $10=$ very easy).

Cognitive testing was undertaken with the Cogstate brief test battery ${ }^{10-14}$ using a computerized playing card interface for the following tasks:

1. Detection ("Has the card turned over?") with speed of response as the outcome

2. Identification ("Is the card red?") with speed of response as the outcome
3. One-card learning ("Have you seen this card before in this task?") with accuracy as the outcome

4. One-back memory ("Is the card the same as the previous card?") with both speed and accuracy as outcomes.

Baseline cognitive testing occurred on the day of the procedure after admission to the endoscopy unit. Postprocedure testing occurred once the patient was assessed by unit nursing staff as meeting hospital discharge criteria. Post-procedure impairment in cognitive function was defined by deterioration in performance from baseline, with each task considered separately.

For our analysis, we defined a number of possible complications a priori along with minor and serious subcategories. ${ }^{15}$ Minor hypoxia was determined if the oxygen saturation was $<90 \%$ and the patient was unresponsive to $>15 \mathrm{sec}$ of jaw thrust, verbal stimulus, or increased oxygen flow. Serious hypoxia was considered if bag-mask ventilation or intubation was required. Minor airway obstruction was diagnosed if noisy breathing or an obstructed respiratory pattern occurred requiring jaw thrust or chin lift. Serious airway obstruction was considered if insertion of an oral or nasopharyngeal airway was required. Minor hypotension was defined as a $20 \%$ decrease in mean blood pressure from pre-procedure baseline, systolic blood pressure $<90 \mathrm{mmHg}$, and/or diastolic blood pressure $<50$ $\mathrm{mmHg}$. Serious hypotension was considered if vasoactive drugs or a fluid bolus was required. Minor bradycardia was defined as a $25 \%$ decrease in heart rate from pre-procedure baseline or a heart rate $<55$ beats $\cdot \mathrm{min}^{-1}$. Serious bradycardia was considered if chronotropic drugs were required or if cardiac arrest occurred. Lastly, minor agitation was defined as patient movement during the procedure that required physical restraint or medication bolus. Serious agitation was considered if it required either interruption or abandonment of the procedure or patient injury.

Two hundred patients were randomized by a computergenerated sequence (www.randomization.com) to receive light (BIS 70-80) or deep (BIS < 60) sedation. Randomization results were concealed in opaque envelopes until after consent was obtained and baseline testing was completed. Patients and study personnel undertaking postprocedure observations were blinded to group allocation. The attending anesthesiologists applied standard monitoring plus a BIS sensor and recorded baseline patient data. Sedation was then commenced with a fentanyl bolus (age $<$ $70 \mathrm{yr}=$ fentanyl $0.7 \mu \mathrm{g} \cdot \mathrm{kg}^{-1}$ up to maximum of $100 \mu \mathrm{g}$; age $\geq 70 \mathrm{yr}=$ fentanyl $0.5 \mu \mathrm{g} \cdot \mathrm{kg}^{-1}$ up to maximum of 75 $\mu \mathrm{g})$ and an effect-site-guided target-controlled propofol infusion using the Schnider model. ${ }^{16}$ Once the BIS target was achieved, the procedure commenced and the propofol 
target concentration was adjusted by the anesthesiologist as required to maintain the BIS within range.

The defined complications during sedation were managed as clinically appropriate and recorded by the anesthesiologist. At the end of the procedure, the endoscopist rated the ease of completion of the colonoscopy on an 11-point Likert scale, and both the endoscopist and anesthesiologist rated satisfaction with sedation on a five-point Likert scale.

In the PACU, routine clinical care was undertaken, and when the patients were alert $(\mathrm{MOAA} / \mathrm{S}=5)$, they were assessed for procedural recall. Once the patients were ready for hospital discharge according to the Chung discharge criteria, ${ }^{17}$ cognitive testing was repeated and patient satisfaction with sedation was recorded.

\section{Statistical analysis}

The incidence of recall during propofol sedation for colonoscopy has been reported to be $3-4 \%$ when a deeper sedation was targeted. ${ }^{18-20}$ Therefore, no recall in $95 \%$ of patients undergoing deep propofol-based sedation was used as the baseline for sample size calculation. An equivalence margin of $10 \%$ in the incidence of recall between the light and deep sedation groups was selected by investigator consensus, given the lack of literature to inform this decision. For a significance level of 0.05 and a power of 0.8 , each group therefore needed 76 participants (total $=$ 152) to show equivalence. A recruitment target of 200 patients was set to account for dropouts and to allow sufficient power for analysis of secondary outcomes.

Data were analyzed on an intention-to-treat basis, except for recall, which was also analyzed on an as-per-protocol basis given the equivalence hypothesis for the primary endpoint.

The BIS data were collected electronically at oneminute intervals on a stand-alone BIS monitor, and the output was downloaded into an Excel spreadsheet. If the signal quality index was $<50$, the data at that time point were excluded. All remaining values from commencement of drug administration to completion of the procedure were included.

Continuous data were tested for normality and then summarized using mean (SD) if normally distributed or median (interquartile range [IQR]) if non-normally distributed. Effect sizes and confidence intervals (CIs) are provided, and CIs for the non-normally distributed data were calculated by the Somers' D Z transformation method. For each Cogstate task, non-normally distributed data were arcsine or log-transformed to permit the use of parametric methods before calculating the mean (SD) of speed of response and accuracy. Categorical data were summarized using number $(\%)$.
The difference in the rate of recall between patients in the light and deep sedation groups was analyzed for equivalence using the two one-sided test (TOST) approach and assessment of the equivalence margin. ${ }^{21}$ With this method, equivalence would be established if the $90 \% \mathrm{CI}$ for the difference between recall rates with light and deep sedation fell within the prespecified $10 \%$ equivalence margin.

Comparisons between the two randomized groups were made using unpaired two-tailed Student's $t$ tests (normally distributed data), Wilcoxon rank-sum tests (non-normally distributed data), and Chi square or Fisher's exact tests (categorical data). Planned analyses included comparison of achieved BIS values, medication dosages, complications, durations, procedural ease, and MOAA/S scores in the PACU. Chi square testing was used to explore the relationship between ASA physical status and the occurrence of complications for the whole cohort and for the light and deep sedation groups. Comparisons between the discharge and baseline cognitive tests were made using paired two-tailed Student's $t$ tests. Analysis of covariance was used to analyze the effect of sedation depth, ASA physical status, and sex on post-procedure cognitive function, with preprocedure cognition also included in the model. The effect of ASA physical status on cognitive recovery was analyzed by analysis of variance. Effect-size measures were used to express the magnitude of differences between (Cohen's d) or within (Dunlap's d) groups for the cognitive testing. For Cohen's d and Dunlap's d, generally an effect-size value $>0.1=$ trivial effect; 0.1 $0.3=$ small effect; $0.3-0.5=$ medium effect; and $>0.5=$ large effect. ${ }^{22} \mathrm{~A}$ reliable change index (RCI) for each patient for the detection task in the Cogstate test battery was calculated to quantify clinically significant cognitive decline. The RCI allows for assessment of impairment from baseline testing at the individual patient level ${ }^{14}$ and has been used to assess post-sedation recovery. ${ }^{20}$ It is calculated by obtaining the task speed at discharge, subtracting the task speed at baseline, and dividing by the within-subject SD. An index $>1$ has been calculated as impairment equivalent to a blood alcohol concentration of $0.05 \% .^{13,14}$ The Kruskal-Wallis rank test was used to compare sedation satisfaction between the two patient groups, the anesthesiologists, and the endoscopists. All recorded $P$ values are two-sided. The majority of the analyses were conducted using STATA $^{\circledR}$ version 12 (Stata Corporation, College Station, TX, USA), with supplementary analysis conducted using SPSS $^{\circledR}$ version 22 (Released 2013, IBM SPSS Statistics for Windows, IBM Corp., Armonk, NY, USA). 


\section{Results}

Of the 300 eligible patients screened for enrolment, 200 were subsequently randomized. Reasons for failure to randomize eligible patients are outlined in Fig. 1. One patient randomized to the light sedation group was mistakenly treated according to the deep sedation group protocol. The group characteristics were well balanced at baseline (Table 1). The median [IQR] BIS during the colonoscopy in patients randomized to light sedation (target BIS 70-80) was 69 [65-74] and in patients randomized to deep sedation (target BIS $<60$ ) was 53 [46-59] $(P<0.001)$ (Table 2 and Fig. 2). Patients in the light sedation group received a significantly lower median [IQR] dose of propofol than patients in the deep sedation group (240 [170-320] $\mathrm{mg}$ vs 326 [250-420] $\mathrm{mg}$, respectively; $P<0.001$ ).

As per our plan for statistical analysis, both the intention-to-treat and the as-per-protocol analyses were performed on the primary outcome. In both instances, the incidence of recall was $12 \%$ in the light sedation group $v s$ $1 \%$ in the deep sedation group. Using the TOST procedure, the risk difference for the per-protocol analysis and for the intention-to-treat analysis was 0.11 (90\% CI, 0.06 to 0.17 ) and 0.11 ( $90 \%$ CI, 0.05 to 0.17 ), respectively. Therefore,

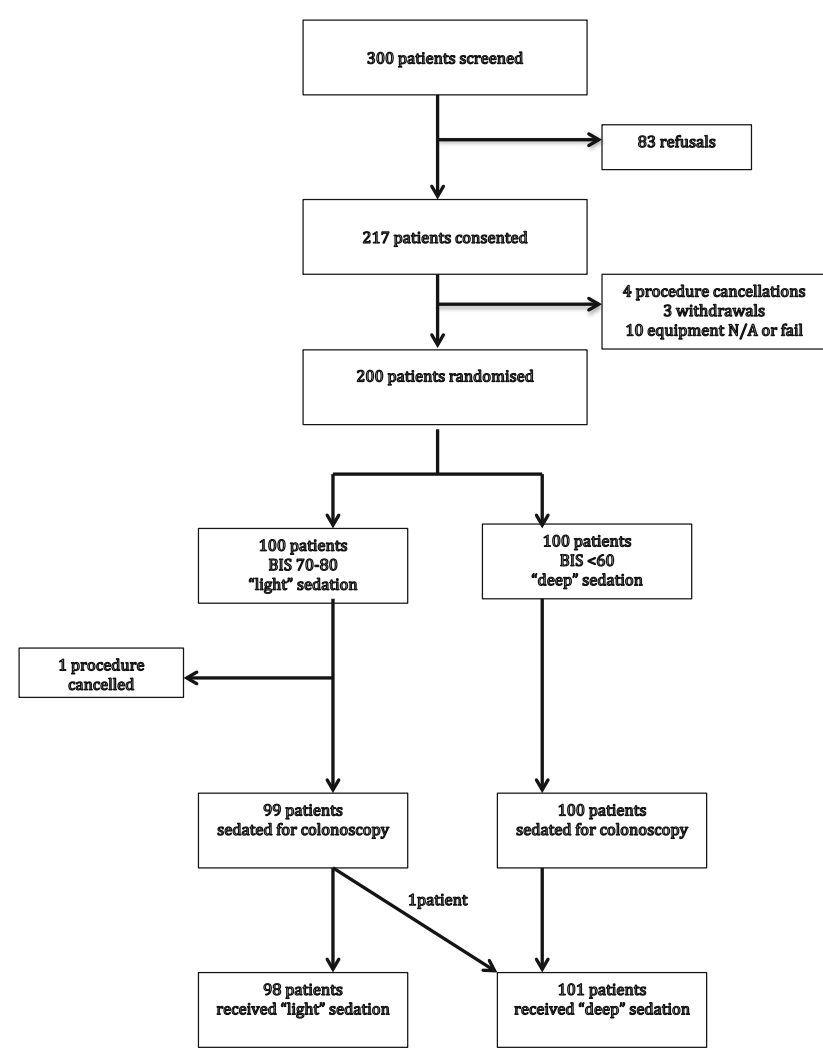

Fig. 1 Study patient flowchart for both strategies equivalence was refuted at the 0.05 significance level with an equivalence margin of $10 \%$.

Sedation and procedure times and ease of procedural completion were similar between groups (Table 2). Nevertheless, patients randomized to deep sedation were significantly more likely to suffer any complication during sedation than patients randomized to light sedation $(66 \% \mathrm{vs}$ $47 \%$, respectively; $P=0.008$ ) (Table 2). Specifically, airway obstruction (28\% vs 14\%, respectively; $P=0.017$ ) and hypotension ( $46 \%$ vs $24 \%$, respectively; $P=0.001$ ) occurred more frequently in patients randomized to deep sedation than in patients randomized to light sedation. In contrast, agitation was more common in patients randomized to light sedation than in patients randomized to deep sedation (22\% vs $11 \%$, respectively; $P=0.033$ ).

There was a low incidence of serious complications and no significant difference between groups (17\%, deep sedation group $v s 14 \%$, light sedation group; $P=0.697$ ). There was no significant relationship between ASA physical status and the incidence of complications in the whole cohort $(P=0.532)$, the light sedation group $(P=$ $0.702)$, or the deep sedation group $(P=0.816)$. Exploratory post hoc analysis in patients with any complication $v$ s those without complications revealed that the median [IQR] BIS was significantly lower (62 [50-68] vs 66 [55-72], respectively; $P=0.010$ ) and the median [IQR] dose of propofol was significantly higher (320 [220-400] mg vs 250 [180-320]mg, respectively; $P<0.001)$.

The satisfaction scores for the anesthesiologists, endoscopists, and patients were all similar regardless whether light or deep sedation was administered (Table 2). Nevertheless, anesthesiologists were significantly less likely than endoscopists or patients to be highly satisfied with light (compared with deep) sedation (53\% vs $79 \%$ vs 97\%, respectively; $P<0.001$ ). In addition, patients reporting recall were less likely to be satisfied with their overall sedation than patients not reporting recall $(10 / 13$ [77\%] vs 185/186 [99\%], respectively; $P<0.001$ ).

Early recovery was more rapid in patients randomized to light sedation than in those randomized to deep sedation: median [IQR] MOAA/S on arrival in the PACU (4 [3-4] vs 2 [0-3], respectively; $P<0.001$ ); mean (SD) time taken to MOAA/S = 5 [3 (4) min vs 7 (4) min, respectively; $P<$ 0.001] (Table 2). There were also significant differences between the light and deep sedation groups in median [IQR] PACU discharge times (18 [15-22] min vs 20 [1725] min, respectively; $P=0.013$ ) and median [IQR] hospital discharge times (65 [57-80] min vs 74 [63-86] min, respectively; $P=0.001$ ) favouring the light sedation group, although the magnitudes of the respective differences (i.e., 2 min and 9 min) were small (Table 2).

Cognitive testing revealed significant post-procedure impairment in the entire study cohort compared with 
baseline. This was reflected in slower psychomotor function and attention (detection and identification tasks) and decreased accuracy of memory (one-card learning task) (Table 3). Nevertheless, there was no difference between the light and deep sedation groups in the magnitude of post-procedure impairment, taking into account pre-procedure performance and age (Table 4). When cognitive deterioration ( $\mathrm{RCI}>1)$ was assessed postprocedure at the individual patient level, 35 patients (18\%) experienced a clinically significant change with no significant difference between those randomized to light and deep sedation (19\% vs $16 \%$, respectively; $P=0.554)$.

\section{Discussion}

Procedural recall rates in adults having elective outpatient colonoscopy with standardized BIS-guided propofol and fentanyl sedation were not equivalent in patients receiving light $v s$ deep sedation. Nevertheless, since our study was designed to test an equivalence hypothesis, no meaningful conclusions can be made regarding the direction of effect of the different rates of recall in the light $v s$ the deep sedation groups. Light sedation was associated with more intraoperative agitation but resulted in fewer cardiorespiratory complications and faster recovery than

Table 1 Patient characteristics

\begin{tabular}{lll}
\hline Characteristic & $\begin{array}{l}\text { BIS 70-80 } \\
(n=99)\end{array}$ & $\begin{array}{l}\text { BIS }<60 \\
(n=100)\end{array}$ \\
\hline Age (yr), mean (SD) & $53(14)$ & $50(16)$ \\
Sex $(\mathrm{male})$ & $54(55)$ & $49(49)$ \\
BMI $\left(\mathrm{kg} \cdot \mathrm{m}^{-2}\right)$, median [IQR] & $27[25-31]$ & $26[23-29]$ \\
ASA physical status & & \\
I & $34(34)$ & $37(37)$ \\
II & $42(42)$ & $48(48)$ \\
III & $23(23)$ & $15(15)$ \\
Medications & $7(7)$ & $7(7)$ \\
Antidepressant & $3(3)$ & $0(0)$ \\
Antipsychotic & $4(4)$ & $2(2)$ \\
Sedative & $2(2)$ & $2(2)$ \\
Opioid & $11(11)$ & $10(10)$ \\
Any of the above & & \\
Baseline Monitoring & $74(14)$ & $73(15)$ \\
Heart rate, mean (SD) & $136(24)$ & $137(22)$ \\
Systolic blood pressure, mean (SD) & $99[98-100]$ & $99[98-100]$ \\
Oxygen saturation, median [IQR] & $98[97-98]$ & $98[95-98]$ \\
BIS, median [IQR] &
\end{tabular}

BIS = bispectral index; ASA = American Society of Anesthesiologists' physical status grade; $\mathrm{BMI}=$ body mass index; IQR = interquartile range. Reported as number (percentage) unless denoted deep sedation. Sedation depth was not a significant predictor of cognitive impairment at hospital discharge, but statistically significant cognitive impairment (albeit mild) was recorded in both groups.

The incidences of recall in our study were consistent with other studies using propofol-based sedation of various depths. ${ }^{20,23,24}$ In studies of deep propofol-based sedation, procedural recall rates of $3-4 \%$ were reported. ${ }^{20,23}$ In contrast, in studies involving light to moderate propofolbased sedation, procedural recall rates of $14-50 \%$ were reported. ${ }^{24}$ Adjuvant drugs varied amongst these studies, and none of the studies were randomized trials. In this novel study, we randomized patients undergoing colonoscopy to either light or deep sedation so postprocedural recall could be compared following propofol and fentanyl-based sedation to a standardized anesthetic depth using BIS.

Patient satisfaction with sedation was high in this and previous studies. ${ }^{20,23,24}$ Most patients who did experience recall were still satisfied with their sedation. Chatman et al. ${ }^{1}$ found that pre-procedure discussion about awareness reduced patients' anxiety regarding this possibility. Therefore, with appropriate discussion and consent, light sedation for colonoscopy may be acceptable to patients despite the higher incidence of recall. In another study, patients undergoing colonoscopy were randomized to either routine general anesthesia or patient-controlled propofol sedation. Results showed that significantly more patients would repeat the procedure with the same anesthetic if they received sedation. ${ }^{25}$ Further, $25 \%$ of patients did not use any sedation for the procedure, ${ }^{25}$ which suggests that deep sedation may not be desired by a large number of patients.

Anesthesiologists were equally satisfied with light or deep sedation, but they were less likely to be satisfied with the sedation than patients and endoscopists. The basis of this lower satisfaction may have been the requirement to administer propofol by target-controlled infusion rather than by intermittent bolus delivery. Bolus delivery is preferred by many sedation providers perhaps because titration is more difficult to perform. The target-controlled infusion of propofol was used as protocol to assist with maintaining a constant depth of sedation and to facilitate modelling of effect-site concentrations.

Light and deep sedation each have advantages and disadvantages that must be balanced against the incidence of recall. The logistic factors were either the same between groups (sedation time, procedure time, procedural ease) or favoured the light sedation group (time until MOAA/S $=5$, time in PACU, and time until hospital discharge). Although the magnitudes of the differences were small, the procedural or financial ramifications may be significant given the large number of colonoscopies performed. The 
Table 2 Sedation, procedure, and recovery characteristics

\begin{tabular}{|c|c|c|c|c|}
\hline Characteristic & $\begin{array}{l}\text { BIS 70-80 } \\
(n=99)\end{array}$ & $\begin{array}{l}\text { BIS }<60 \\
(n=100)\end{array}$ & $\begin{array}{l}\text { Effect size and } \\
95 \% \text { Confidence Interval }\end{array}$ & $P$ value \\
\hline Fentanyl dose $(\mu \mathrm{g})$, mean (SD) & $60(13)$ & $57(14)$ & $-3.17(-6.92$ to 0.57$)$ & 0.096 \\
\hline Total propofol dose $(\mathrm{mg})$ & $240[170-320]$ & $326[250-420]$ & $-43 \%(-56$ to -28$)$ & $<0.001$ \\
\hline Propofol infusion dose $\left(\mathrm{mg} \cdot \mathrm{min}^{-1}\right)$ & $12[9-14]$ & 15 [13-19] & $-53 \%(-65$ to -39$)$ & $<0.001$ \\
\hline Median BIS & $69[65-74]$ & $53[46-59]$ & $81 \%(70$ to 88$)$ & $<0.001$ \\
\hline Proportion of time BIS in target range & $18[4-35]$ & $43[6-78]$ & $-28 \%(-45$ to -11$)$ & 0.001 \\
\hline Any Complication & $47(47 \%)$ & $66(66 \%)$ & $-0.19(-0.32$ to -0.05$)$ & 0.010 \\
\hline Any Serious Complication & $14(14 \%)$ & $17(17 \%)$ & $-0.03(-0.13$ to 0.07$)$ & 0.697 \\
\hline \multicolumn{5}{|l|}{ Hypoxia } \\
\hline Present & $0(0)$ & $4(4 \%)$ & $-0.04(-0.08$ to -0.00$)$ & 0.121 \\
\hline Serious & $0(0)$ & $1(1 \%)$ & $-0.01(-0.03$ to 0.01$)$ & 1.00 \\
\hline \multicolumn{5}{|l|}{ Airway } \\
\hline Present & $14(14 \%)$ & $28(28 \%)$ & $-0.14(-0.25$ to -0.03$)$ & 0.017 \\
\hline Serious & $2(2 \%)$ & $2(2 \%)$ & $0.00(-0.04$ to 0.04$)$ & 1.000 \\
\hline \multicolumn{5}{|l|}{ Hypotension } \\
\hline Present & $24(24 \%)$ & $46(46 \%)$ & $-0.22(-0.35$ to -0.09$)$ & 0.001 \\
\hline Serious & $9(9 \%)$ & $14(14 \%)$ & $-0.05(-0.14$ to 0.04$)$ & 0.279 \\
\hline \multicolumn{5}{|l|}{ Bradycardia } \\
\hline Present & $3(3 \%)$ & $1(1 \%)$ & $0.02(-0.02$ to 0.06$)$ & 0.369 \\
\hline Serious & $0(0)$ & $0(0)$ & - & - \\
\hline \multicolumn{5}{|l|}{ Agitation } \\
\hline Present & $22(22 \%)$ & $11(11 \%)$ & $0.11(0.01$ to 0.21$)$ & 0.033 \\
\hline Serious & $3(3 \%)$ & $1(1 \%)$ & $0.02(-0.02$ to 0.06$)$ & 0.369 \\
\hline Sedation time (min), mean (SD) & $24(12)$ & $24(12)$ & $0.56(-2.79$ to 3.92$)$ & 0.741 \\
\hline Procedure time (min), mean (SD) & $19(11)$ & $19(12)$ & $<-0.01(-3.27$ to 3.27$)$ & 1.000 \\
\hline Ease of procedure completion $\dagger$ & 7 [5-9] & $8[6-9]$ & $-14 \%(-29$ to 2$)$ & 0.084 \\
\hline \multicolumn{5}{|l|}{ Sedation Satisfaction } \\
\hline Endoscopists highly satisfied & $79(79 \%)$ & $80(80 \%)$ & $-0.01(-0.12$ to 0.10$)$ & 1.000 \\
\hline Anesthesiologist highly satisfied & $53(53 \%)$ & $59(59 \%)$ & $-0.06(-0.20$ to 0.08$)$ & 0.474 \\
\hline Patient highly satisfied & $96(97 \%)$ & $99(99 \%)$ & $-0.02(-0.06$ to 0.02$)$ & 0.369 \\
\hline $\mathrm{MOAA} / \mathrm{S}$ on arrival to $\mathrm{PACU}$ & $4[3-4]$ & $2[0-3]$ & $63 \%$ (49 to 74$)$ & $<0.001$ \\
\hline Time until OAA/S = 5, mean $(\mathrm{SD})$ & $3(4)$ & $7(4)$ & $3.23(2.19$ to 4.27$)$ & $<0.001$ \\
\hline Time in PACU & $18[15-22]$ & $20[17-25]$ & $3.03(0.93$ to 5.12$)$ & 0.013 \\
\hline Time from start of sedation until PACU discharge, mean (SD) & $45(14)$ & $49(15)$ & $3.71(-0.46$ to 7.88$)$ & 0.081 \\
\hline Time from start of sedation until hospital discharge, mean (SD) & $65(57-80)$ & $74(63-86)$ & $-26 \%(-41$ to -11$)$ & 0.001 \\
\hline Recall & $12(12 \%)$ & $1(1 \%)$ & $0.11(0.05 \text { to } 0.17)^{\wedge}$ & 0.001 \\
\hline
\end{tabular}

BIS = bispectral index. Time reported in minutes. MOAA/S = modified observer's assessment of alertness $/$ sedation $;{ }^{9} \mathrm{PACU}=$ postanesthesia care unit. Reported as median [interquartile range] unless denoted. $\dagger$ Scored on $0-10$ scale with $0=$ impossible and $10=$ very easy. Satisfaction measured on five-point Likert scale from $1=$ very dissatisfied to $5=$ very satisfied. For sedation satisfaction those highly satisfied $=$ number $(\%)$ who scored 4 or 5 on Likert scale. The effect sizes for normally distributed data are mean difference (95\% confidence interval). For non-normal data, the effect sizes are calculated by Somers' D methods where the effect of being in the light compared with the deep sedation group is reported as percentage chance (95\% confidence interval). This can be interpreted as the percentage chance that a patient randomized to light $v s$ deep sedation will have a higher value for the parameter (lower percentage likelihood if negative). For categorical data risk difference, the $95 \%$ confidence interval is reported

incidence of complications was either not different between groups (hypoxia, bradycardia) or dependent on the group. Agitation was more common in the light sedation group, while hypotension and airway obstruction were common in the deep sedation group. Progression to serious consequences was rare, however, and not different between groups. Given our limited sample size, the conclusions relating to complications for endoscopy sedation are guarded, but our findings are consistent with other studies. ${ }^{15,19,25-28}$ 
Fig. 2 Bispectral index (BIS) data for each patient. Trend lines for light sedation (BIS 70-80) and deep sedation (BIS $<60$ ) group medians at each time point with capture at oneminute intervals

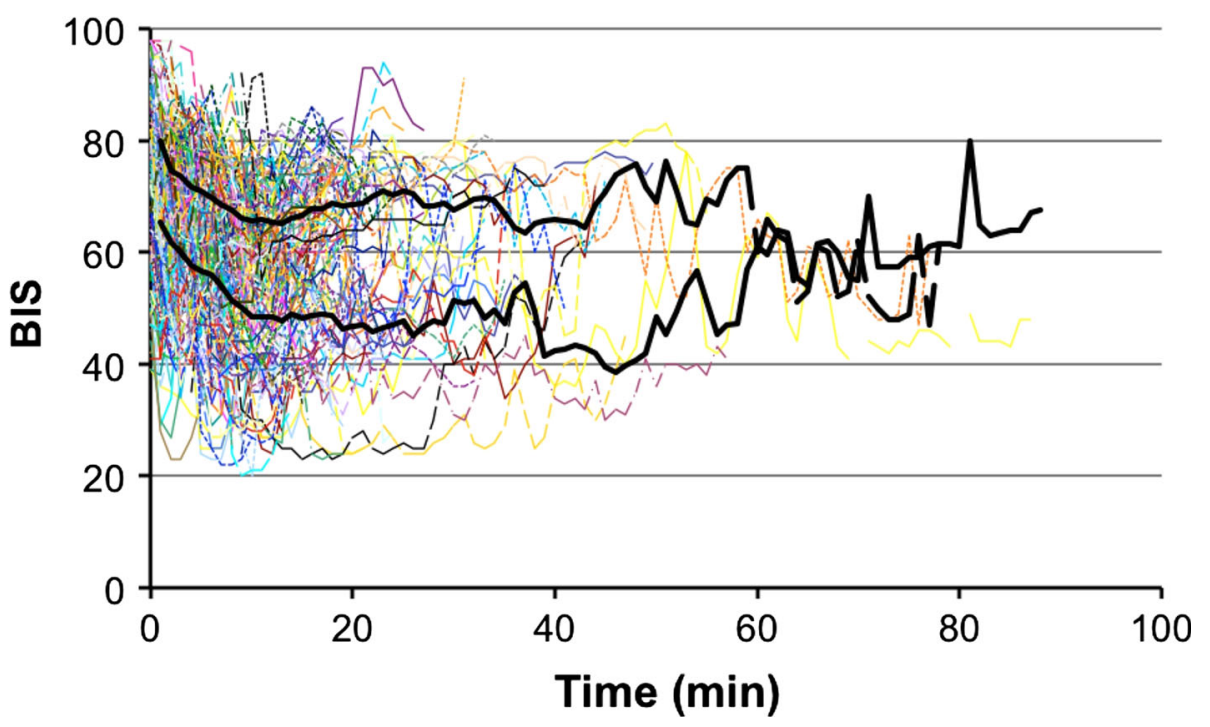

Table 3 Cognitive testing pre and post procedure

\begin{tabular}{llllrl}
\hline Characteristic & $n$ & Pre-procedure & Post-procedure & $P$ value & Effect size (Dunlap's d) \\
\hline Identification $\left(\log _{10} \mathrm{msec}\right)$ & 196 & $2.76(0.11)$ & $2.79(0.11)$ & $<0.001$ & -0.48 \\
Detection $\left(\log _{10} \mathrm{msec}\right)$ & 196 & $2.58(0.13)$ & $2.60(0.13)$ & 0.004 & -0.16 \\
One-card learning (arcsine accuracy) & 196 & $0.96(0.11)$ & $0.92(0.12)$ & $<0.001$ & 0.36 \\
One-back memory (arcsine accuracy) & 194 & $1.24(0.27)$ & $1.29(0.27)$ & 0.004 & -0.16 \\
One-back memory $\left(\log _{10} \mathrm{msec}\right)$ & 192 & $2.96(0.12)$ & $2.95(0.11)$ & 0.040 & 0.098 \\
\hline
\end{tabular}

Pre- and post-procedure performance reported as mean $(\mathrm{SD}) . \log _{10} \mathrm{msec}=$ milliseconds $\log$ transformed; arcsine accuracy $=$ proportion correct arcsine transformed

Cogstate brief test battery: Identification task ("Is the card red?") with primary outcome of speed of response; Detection task ("Has the card turned over?") with primary outcome of speed of response; One-card learning ("Have you seen this card before in this task?") with primary outcome of accuracy; One-back memory ("Is the card the same as the previous card?") with outcomes of both speed and accuracy. Slower response times and reduced accuracy are indicative of impairment. For Dunlap's d effect size, a value $>0.1=$ trivial effect; $0.1-0.3=$ small effect; $0.3-0.5=$ medium effect; $>0.5=$ large effect. ${ }^{22}$ Positive effect size indicates improved performance post sedation. Negative effect size indicates poorer performance in post-procedure than in pre-procedure testing

Table 4 Cognitive testing by sedation depth

\begin{tabular}{|c|c|c|c|c|}
\hline Task & BIS 70-80 & $\mathrm{BIS}<60$ & $P$ value & Effect Size (Cohen's d) \\
\hline Identification $\left(\log _{10} \mathrm{msec}\right)$ & $2.79(0.01)$ & $2.79(0.01)$ & 0.726 & 0 \\
\hline Detection $\left(\log _{10} \mathrm{msec}\right)$ & $2.60(0.01)$ & $2.60(0.01)$ & 0.803 & 0 \\
\hline One-card learning (arcsine accuracy) & $0.92(0.01)$ & $0.92(0.01)$ & 0.973 & 0 \\
\hline One-back memory (arcsine accuracy) & $1.29(0.02)$ & $1.29(0.02)$ & 0.800 & 0 \\
\hline One-back memory $\left(\log _{10} \mathrm{msec}\right)$ & $2.94(0.01)$ & $2.95(0.01)$ & 0.265 & -0.095 \\
\hline
\end{tabular}

Cogstate tasks reported as mean (standard error at median age). The model includes pre-procedure cognitive testing performance, American Society of Anesthesiologists' physical status, and sex. $\log _{10} \mathrm{msec}=$ milliseconds log transformed; arcsine accuracy = proportion correct arcsine transformed

Cogstate brief test battery: Identification task ("Is the card red?") with primary outcome of speed of response; Detection task ("Has the card turned over?") with primary outcome of speed of response; One-card learning ("Have you seen this card before in this task?") with primary outcome of accuracy; One-back memory ("Is the card the same as the previous card?") with outcomes of both speed and accuracy. Slower response times and reduced accuracy are indicative of impairment. For Cohen's d effect size, a value $>0.1=$ trivial effect; $0.1-0.3=$ small effect; 0.3-0.5 = medium effect; $>0.5=$ large effect. ${ }^{22}$ Positive effect size indicates improved performance post sedation. Negative effect size indicates poorer performance in post-procedure than in pre-procedure testing 
At hospital discharge, patients in this study were cognitively impaired with reduced speed of response and task accuracy, as measured by the Cogstate brief test battery, which is similar to previous studies. ${ }^{10-14}$ These results did not differ between patients with light $v s$ deep sedation; consequently, while light sedation might confer logistic advantages, they cannot be extended to cognitive improvement at hospital discharge. Patients were not retested; thus, no conclusions can be made between the light and deep sedation groups regarding the speed or completeness of recovery to baseline cognition. This would be an interesting extension to this work to investigate in the future. Nevertheless, this novel study uses a validated assessment tool to determine cognitive impairment after standardized light and deep sedation.

This study has several limitations. The number of patients is too small to assess rare complications and does not include longer term cognitive testing or assessment of extended postoperative recall. The baseline cognitive testing was performed on the day of the colonoscopy when patients were fasting, had received bowel preparation solution, and may have been anxious. It is possible that these conditions may have adversely impacted on baseline performance and that post-procedure impairment was underestimated as a result. In addition, the sedation profile of 'light' and 'deep' throughout each case does not coincide with the common practice of providing deep sedation at the start of the case - to facilitate transit of the sigmoid colon - with gradual lightening as the endoscope is removed.

This study is important because it compares clinically relevant light and deep sedation for meaningful outcomes, including recall, recovery times, and complications, including intraprocedural complications and cognitive impairment at discharge. The sedative medications used in this study reflect mainstream practice for many sedation providers, and the broad inclusion criteria make the findings relevant to a general endoscopy population. The findings from this study can inform the discussion between sedation providers and their patients regarding the risks and benefits of endoscopy sedation.

Funding Australian and New Zealand College of Anaesthetists Project Grant (12/021).

Conflict of interest Paul Maruff is an employee of Cogstate Ltd, the company that provided the Cogstate test battery used in this study.

\section{References}

1. Chatman N, Sutherland JR, van der Zwan R, Abraham N. A survey of patient understanding and expectations of sedation/ anaesthesia for colonoscopy. Anaesth Intensive Care 2013; 41: 369-73.

2. McLachlan SA, Clements A, Austoker J. Patients' experiences and reported barriers to colonoscopy in the screening context - a systematic review of the literature. Patient Educ Couns 2012; 86: $137-46$.

3. Denberg TD, Melhado TV, Coombes JM, et al. Predictors of nonadherence to screening colonoscopy. J Gen Intern Med 2005; 20: 989-95.

4. Johansen JW, Sebel PS. Development and clinical application of electroencephalographic bispectrum monitoring. Anesthesiology 2000; 93: 1336-44.

5. Barr G, Anderson RE, Owall A, Jakobsson JG. Being awake intermittently during propofol-induced hypnosis: a study of BIS, explicit and implicit memory. Acta Anaesthesiol Scand 2001; 45: 834-8.

6. Baker GW, Sleigh JW, Smith P. Electroencephalographic indices related to hypnosis and amnesia during propofol anaesthesia for cardioversion. Anaesth Intensive Care 2000; 28: 386-91.

7. Brice DD, Hetherington RR, Utting JE. A simple study of awareness and dreaming during anaesthesia. Br J Anaesth 1970; 42: 535-42.

8. Abouleish E, Taylor FH. Effect of morphine-diazepam on signs of anesthesia, awareness, and dreams of patients under $\mathrm{N} 2 \mathrm{O}$ for cesarean section. Anesth Analg 1976; 55: 702-5.

9. Chernik DA, Gillings D, Laine H, et al. Validity and reliability of the Observer's Assessment of Alertness/Sedation Scale: study with: intravenous midazolam. J Clin Psychopharmacol 1990; 10: 244-51.

10. Collie A, Darekar A, Weissgerber G, et al. Cognitive testing in early-phase clinical trials: development of a rapid computerized test battery and application in a simulated Phase I study. Contemp Clin Trials 2007; 28: 391-400.

11. Falleti $M G$, Maruff $P$, Collie A, Darby DG. Practice effects associated with the repeated assessment of cognitive function using the CogState battery at 10-minute, one week and one month test-retest intervals. J Clin Exp Neuropsychol 2006; 28: 1095-112.

12. Fredrickson A, Snyder PJ, Cromer J, Thomas E, Lewis M, Maruff $P$. The use of effect sizes to characterize the nature of cognitive change in psychopharmacological studies: an example with scopolamine. Hum Psychopharmacol 2008; 23: 425-36.

13. Maruff P, Falleti MG, Collie A, Darby D, McStephen M. Fatiguerelated impairment in the speed, accuracy and variability of psychomotor performance: comparison with blood alcohol levels. J Sleep Res 2005; 14: 21-7.

14. Maruff P, Werth J, Giordani B, Caveney AF, Feltner D, Snyder $P J$. A statistical approach for classifying change in cognitive function in individuals following pharmacologic challenge: an example with alprazolam. Psychopharmacology (Berl) 2006; 186: 7-17.

15. Lee CK, Lee SH, Chung IK, et al. Balanced propofol sedation for therapeutic GI endoscopic procedures: a prospective, randomized study. Gastrointest Endosc 2011; 73: 206-14.

16. Schnider TW, Minto CF, Shafer SL, et al. The influence of age on propofol pharmacodynamics. Anesthesiology 1999; 90: 1502-16.

17. Chung F. Discharge criteria - a new trend. Can J Anaesth 1995; 42: $1056-8$.

18. Eer ASY, Padmanabhan U, Leslie K. Propofol dose and incidence of dreaming during sedation. Eur J Anaesthesiol 2009; 26: 833-6.

19. Imagawa A, Fujiki $S$, Kawahara $Y$, et al. Satisfaction with bispectral index monitoring of propofol-mediated sedation during endoscopic submucosal dissection: a prospective, randomized study. Endoscopy 2008; 40: 905-9.

20. Padmanabhan U, Leslie K, Eer ASY, Maruff P, Silbert BS. Early cognitive impairment after sedation for colonoscopy: the effect of 
adding midazolam and/or fentanyl to propofol. Anesth Analg 2009; 109: 1448-55.

21. Walker E, Nowacki AS. Understanding equivalence and noninferiority testing. J Gen Intern Med 2011; 26: 192-6.

22. Cohen J. Statistical Power Analysis for the Behavioral Sciences. NY: Academic Press; 1969.

23. Stait ML, Leslie K, Bailey R. Dreaming and recall during sedation for colonoscopy. Anaesth Intensive Care 2008; 36: 685-90.

24. VanNatta ME, Rex DK. Propofol alone titrated to deep sedation versus propofol in combination with opioids and/or benzodiazepines and titrated to moderate sedation for colonoscopy. Am J Gastroenterol 2006; 101: 2209-17.

25. Crepeau T, Poincloux L, Bonny C, et al. Significance of patientcontrolled sedation during colonoscopy: results from a prospective randomized controlled study. Gastroenterol Clin Biol 2005; 29: 1090-6.
26. Australian and New Zealand College of Anaesthetists. Guidelines on Sedation and/or Analgesia for Diagnostic and Interventional Medical, Dental or Surgical Procedures (PS09). 2014. Available from URL: http://www.anzca.edu.au/resources/professional-docum ents/pdfs/ps09-2014-guidelines-on-sedation-and-or-analgesia-fordiagnostic-and-interventional-medical-dental-or-surgical-procedures. pdf (accessed June 2015).

27. Correia LM, Bonilha DQ, Gomes GF, et al. Sedation during upper GI endoscopy in cirrhotic outpatients: a randomized, controlled trial comparing propofol and fentanyl with midazolam and fentanyl. Gastrointest Endosc 2011; 73(45-51): e1.

28. Qadeer MA, Vargo JJ, Patel S, et al. Bispectral index monitoring of conscious sedation with the combination of meperidine and midazolam during endoscopy. Clin Gastroenterol Hepatol 2008; 6: 102-8. 